\title{
FORMED ELEMENTS IN THE HUMAN LENS CAPSULE* WITH SPECIAL REFERENCE TO THEIR ALLEGED ROLE IN THE AETIOLOGY OF SENILE CATARACT
}

BY

\author{
A. J. DARK \\ Nuffield Laboratory of Ophthalmology and Oxford Eye Hospital
}

THE lens capsule has long been regarded as a homogeneous 'glass' or hyaline membrane with no discernible microscopic structure, although certain observations, for example, that of Salzmann (1912), who noted step-like fissures at the sites of rupture in the capsule, had led some earlier investigators to consider that it might have a stratified architecture. The recent advent of two new histological procedures-phase-contrast and electron microscopy-has shown that a fine lamellar structure is indeed present (Grignolo, 1954; Koch, 1957).

Apart from its role in accommodation, the lens capsule has a wellestablished function as a filter, preventing the passage to-and-fro of the larger protein molecules between the aqueous and lens epithelium, but permitting the passage of smaller ones (Friedenwald, 1930; Pirie and van Heyningen, 1956; François and Rabaey, 1958). It is therefore of interest that no pores or pre-formed pathways were noted in studies with these newer methods of examination until Monahan (1953), using phase-contrast microscopy, described fine channels in the anterior portion of the human lens capsule. The channels begin on the aqueous-bound surface of the anterior capsule in an annular zone some $3 \mathrm{~mm}$. from the anterior pole of the lens, and then run a tortuous course to open on the inner surface of the capsule in the region [pre-equatorial] of the cells of the germinative zone of the capsular epithelium; no channel extends beyond the equator, nor is any present in the posterior capsule. Impressed by their connexion with this zone of the lens epithelium, Monahan believed that these fine canaliculi were, in fact, the yet undiscovered pores in the capsular 'sieve'. He considered that some support for this concept was provided by the results of animal experiments (although the channels could not actually be demonstrated microscopically in any of the animals he examined) in which various aniline dyes were injected into the anterior chamber. In some cases the lens became cataractous and Monahan presumed that channels had been blocked by deposits of the dyes. Further observations which will be mentioned later led Monahan to attribute to these structures an important role in the aetiology of senile cataract. It was not possible to demonstrate the channels by routine histological staining techniques.

The channels in the lens capsule described by Monahan have not been confirmed by any subsequent investigator, at least as far as Monahan (1958)

* Received for publication May 20, 1960. 
and I are aware, which is surprising in view of the obvious importance of his claims. The following study was therefore undertaken, and investigation of the structure of the lens capsule with phase-contrast microscopy has shown fine filaments which appear to correspond to the "channels" described by Monahan. A variety of histological and histochemical procedures has been used to explore the nature of these structures and to find out whether they could be rendered visible independently of the phase-contrast method. The capsules from senile cataractous lenses have been specially studied to determine whether these filaments undergo any change which could be related to the formation of cataracts. Finally, an experiment designed to determine the possible role of artefact in the production of the filaments is described.

\section{Material and Methods}

Material.-Human lenses were obtained from three sources. The lenses from four eyes removed for malignant tumour of the posterior ocular segment (the anterior segment being apparently unaffected) were obtained soon after enucleation. Thirty lenses with varying degrees of opacity were available after intracapsular cataract extraction. Autopsy material comprising ten healthy lenses was obtained at varying times after death (6-18 hrs). Sections from the lenses of a reputedly adult rhesus monkey (Macaca mulatta) and of four adult rabbits were also studied.

Methods.-The fixatives used included 10 per cent. neutral formalin, 10 per cent. calcium formol, Bouin's fluid, and Palade's buffered osmium tetraoxide (Palade, 1952). Frozen sections of the lens were cut from calcium-formol fixed material. Celloidin sections were prepared from the anterior segments of eyes enucleated for malignant choroidal tumours; flat whole-mount preparations of the capsules of fixed and unfixed lenses were also used. Lens capsules fixed in Palade's solution were embedded in methyl methacrylate and sections 0.5 to $2 \mu$ thick were cut with a glass knife.

For general histological examination sections and whole-mount preparations of lens capsule were stained with haematoxylin and eosin; Mallory's trichrome stain, van Gieson's method, the orcein stain for elastin, and Gordon and Sweet's method for reticulin.

For investigation of the nature of the capsular filaments, a variety of histological and histochemical methods was used. These techniques will be referred to with the results obtained.

A Zeiss-Opton microscope was used for ordinary microscopy, for phase-contrast examination, and for examination in polarized light. Fixed whole-mount preparations and sections were submitted to fluorescence microscopy using ultraviolet light of 3,650 $\AA$.

\section{Observations}

Phase-contrast Microscopy.-Observations using phase-contrast were made on meridional celloidin sections (some of the sections were affixed to slides and the celloidin removed), and flat whole-mount preparations of 
human and animal lens capsules. A few thin meridional sections of methacrylate-embedded human material were also examined. Preparations were mounted in normal saline.

In meridional celloidin sections of human material many fine dark striae and dots are visible in the otherwise homogeneous anterior lens capsule. They are seen only in a specific annular zone 1-2 mm. wide, immediately in front of the equator. When viewed in cross-section, or "end on", the striae appear as minute black dots. The distribution of these structures is not entirely uniform; they are most numerous and occupy the full thickness of the capsule towards the equator, ceasing with remarkable abruptness at the equator itself. On the other hand they fade away gradually towards the anterior pole, often disappearing from the inner portion first so that the last striae seen are immediately underneath the pericapsular membrane or zonular lamella, which is itself devoid of structure.

In flat whole-mount preparations, numerous unconnected dark striae or filaments are found in the annular zone of the anterior lens capsule described above. They are 3-20 $\mu$ long and about $0 \cdot 3 \mu$ wide. The filaments taper gradually to fine points. Many of them are sickle-shaped, a few are circular or U-shaped, and occasional filaments undergo a single bifurcation. The filaments are orientated with their long axes parallel to the capsular surfaces, but randomly to each other-hence the parallel striae and dots seen in truly meridional sections.

Many of these features can be seen in Fig. 1 and Fig. 2 (opposite), which, however, illustrate preparations specially stained to show the filaments.

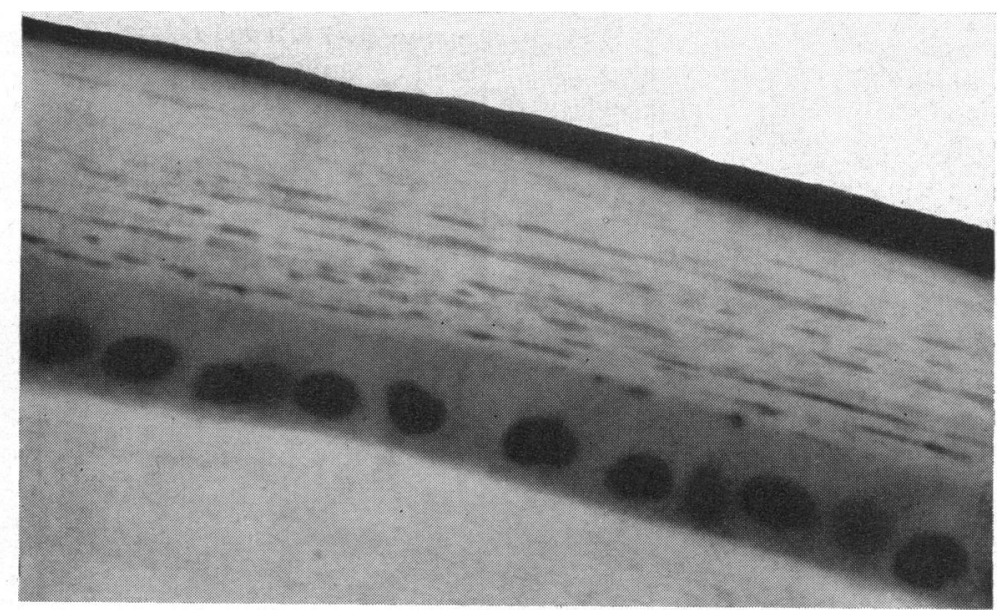

FIG. 1.-Meridional celloidin section through a zone of normal human lens capsule and subcapsular epithelium, just in front of the equator. The thick black surface band corresponds with the orbiculo-anterior capsular fibres of the suspensory zonule. Filaments described in the text lie in the hyaline capsule. They are seen as fine black striae and dots, depending on whether they are viewed longitudinally or in cross-section. Chromehaematoxylin (Gomori). $\times 1,300$. 


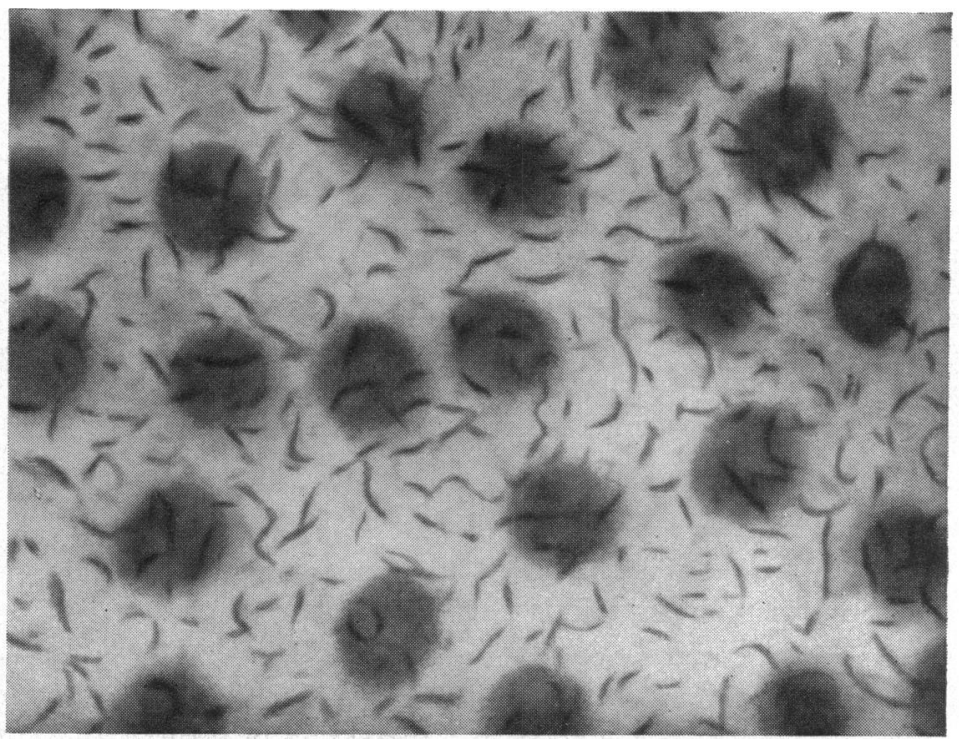

FiG. 2.-Flat whole-mount preparation of normal human lens capsule and subcapsular epithelium obtained from a zone lying just in front of the equator. Filaments illustrated above and described in the text are in sharp focus (epithelial cell nuclei lying at a deeper level are outside the focus and are therefore blurred). The filaments are orientated in random fashion. Chrome-haematoxylin (Gomori). $\quad \times 1,300$.

Under high magnification $(\times 1,300)$ the fanned-out fibrils of the orbiculoanterior capsular fibres are seen lying in a plane distinct from that of the filaments-there is no discernible connexion between these fibrils and the filaments. In places, especially near the cut edges of the tissue, the subcapsular epithelium has occasionally become dislodged during preparation; in such denuded areas the capsular filaments are present in undiminished number and size, an indication that they are located exclusively within the hyaline capsule.

In both flat preparations and meridional sections, the filaments, viewed longitudinally, are seen as dark homogeneous threads; in cross-section, they appear as minute black dots-so that there is no evidence of tubular structure, but rather of solid filaments. This is confirmed by observations on methacrylate-embedded capsular material (after removal of methacrylate): the filaments appear as solid homogeneous threads and dots in between the fine lamellae of the capsule. The lamellar structure of the capsule is discernible when thin $0.5 \mu$ sections are examined with phase-contrast.

It seems possible that the filaments become more numerous with age; the lens of a child aged 6 years contained relatively few filaments, and none was seen in the lens of a 6 months' old infant.

Apart from the infant lens mentioned above, and two other adult lenses which were somewhat autolysed post-mortem specimens, filaments have been found in all normal lenses examined. 
Filamentary structures were not seen in the capsules of any animal lenses studied.

General Histology.-The capsular filaments are not demonstrated by most routine histological methods, Mallory's connective tissue stain, which colours the filaments a pale blue, being an exception. The zonular fibres are also stained blue by this method.

\section{Histochemistry}

Special Microscopy.-The filaments are monorefringent in polarized light, and do not exhibit fluorescence in ultraviolet light of 3,650 $\AA$.

ProteIN: Millon's reagent for tyrosine-containing protein imparts a faint orange colour to the filaments, scarcely distinguishable from that of the capsule.

LIPID: Staining with Sudan Black B does not reveal any lipid. The acidhaematin method for phospholipid is also negative.

CARBOHYDRATE: It is well known that the lens capsule is rich in polysaccharide (Krause, 1933) and that it gives an intense magenta-scarlet colour with the periodic acid-Schiff (PAS) technique of Hotchkiss (Day, 1950) in sections. The filaments are not seen in ordinary sections stained in this way, but in thin methacrylate sections (of a thickness approximating to the filament width) the filaments can be seen as distinct entities, in spite of the fact that they are stained in a pink shade similar to that of the surrounding capsule.

These results indicate that the filaments (like the capsule itself) contain a protein-polysaccharide complex. The possibility that this compound might be an acid mucopolysaccharide was next investigated.

ACID MuCOPOlySACCHARIDE: After staining with 1 per cent. toluidine blue for $1 \mathrm{hr}$ some of the filaments are faintly coloured. It is difficult to be sure about the colour values in such minute structures, but they appear blue rather than red. Incubation of fixed sections with hyaluronidase (Benger) 300 units $/ \mathrm{ml}$. for $3 \mathrm{hrs}$ did not affect this staining. Several techniques, all of which may be used to demonstrate acid mucopolysaccharides, were next used. They included Gram's stain, mucihaematin, mucicarmine, Gomori's aldehyde fuchsin, Alcian blue 8G.S, and the Rinehart-Abủl-Haj method. All gave negative results.

BASOPHILIA: The weak basophilia of the filaments exhibited with toluidine blue can be accentuated by prior oxidation of the sections with periodic acid or permanganate. Presumably more acidic groupings are formed by oxidation. The first step of Gomori's chrome haematoxylin method (Gomori, 1941) embodying these principles shows the filaments clearly. As shown in Fig. 1, the zonular fibres are also stained black; however no connexion could be seen between the zonular fibres and the filaments.

\section{Capsular Filaments and Senile Cataract}

The majority of the capsules from thirty lenses with varying degrees and types of senile cataract were studied by phase-contrast, and also with the staining method just described. 
Capsular filaments were present in all lenses examined (Fig. 3); they were similar in form and distribution and apparently just as densely aggregated as those seen in transparent lenses of a similar age group. Three lenses were hypermature; serial sections of one of them show filaments of normal appearance in every section.

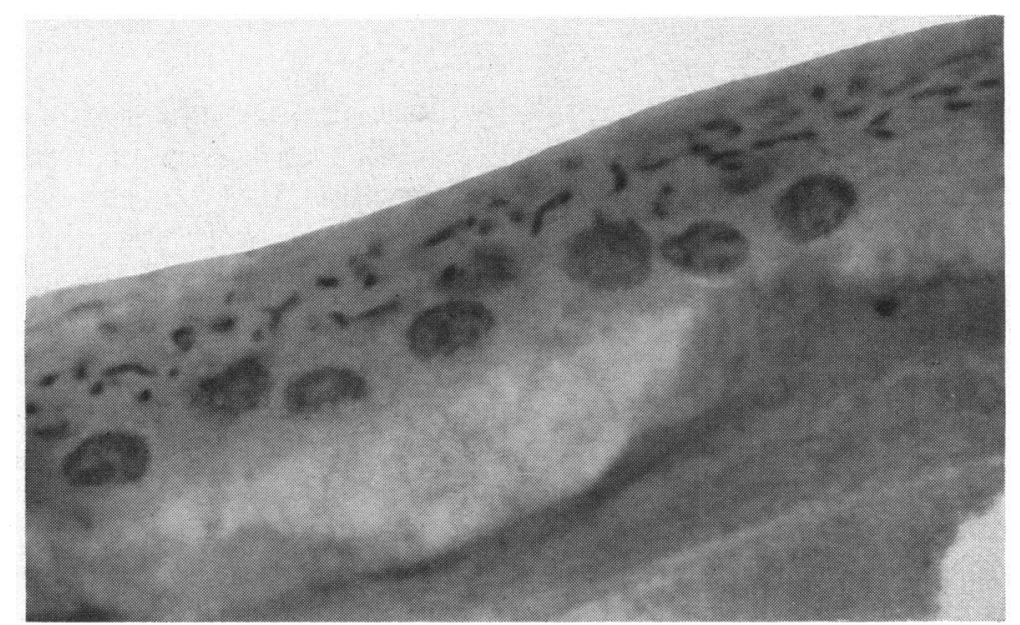

FIG. 3.-Meridional celloidin section of mature senile cataract, showing an abundance of capsular filaments. The capsule is thinner than in a normal lens, probably because of stretching by intumescent lens matter. Chrome-haematoxylin (Gomori). $\times 1,300$.

In a few instances it was noticed, when making flat preparations, that cataractous lens matter remained firmly adherent to the capsular epithelium. Phase-contrast microscopy-as might be anticipated-does not reveal capsular filaments in such thick preparations. However, if these capsules are now stained with the method given above, filaments which are entirely normal in appearance and number become readily visible.

It is concluded that the capsular filaments undergo no change in number or morphology that can be correlated with the genesis of senile cataract.

\section{Are the Capsular Filaments an Artefact?}

The question of artefact naturally arises in any new morphological finding, and probably no tissue is more prone to artefact than the crystalline lens. The following experiment was designed to obviate as many factors conducive to artefact as possible.

Three opaque human lenses were placed in fresh pooled ox aqueous humour at room temperature immediately after removal from patients by the intracapsular extraction technique. The capsules were dissected under aqueous humour. Appropriate flat preparations were then mounted in aqueous humour and cover slips ringed with Vaseline. Under phase-contrast, filaments corresponding exactly in morphology and situation to the description given above for fixed materials 
were readily seen in preparations from the three lenses. The time between placing the lens in the aqueous humour and discovery of the filaments was not more than 10 minutes.

This experiment excludes the production of artefact by fixation, it also appears to eliminate post-mortem change and the gross milieu alterations by the synthetic mountants previously used.

\section{Comment}

Examination of human lens capsule by phase-contrast microscopy demonstrates a striking aggregation of fine thread-like structures in a restricted zone of the anterior lens capsule. This observation, first made by Monahan (1953), is amply confirmed by the present study. In other ways the present observations diverge considerably from Monahan's findings. The structures in question are described in the present study as solid filaments about 3 to $20 \mu$ long; Monahan saw them as fine tortuous channels some 2 or $3 \mathrm{~mm}$. long.

This striking discrepancy in observations may be due to difficulties inherent in phase-contrast microscopy. When thick tissue sections are examined by this method diffraction rims often make it very difficult to interpret the true nature of surfaces and interfaces; in these circumstances specific staining methods may afford more precise information. Furthermore, Monahan did not examine flat whole-mount preparations, such as that illustrated in Fig. 2. Such preparations leave no doubt as to the definitive length of these structures.

Grignolo (1954) and Garzino (1954), both using phase-contrast, looked specifically for the "channels" described by Monahan, but were unable to find them or indeed any comparable structure.

Neither Monahan nor I was able to find these structures in animal lenses.

These filaments cannot be demonstrated satisfactorily with the usual histological stains. Histochemical tests show that they contain a basophilic protein-polysaccharide complex.

Wislocki (1952) noted that zonular fibres of the rhesus monkey (Macaca mulatta) were stained black by the Gomori chrome-haematoxylin method, although the hyaline capsule and the pericapsular membrane (zonular lamella) did not stain with chrome haematoxylin. McCulloch (1954) and Gifford (1958) have subsequently used this technique on the human lens to demonstrate the zonular fibres. Capsular filaments were not noted by these or subsequent investigators.

Capsular filaments are found in a zone of the capsule roughly corresponding with the attachment of the anterior zonular fibres. The possibility that the capsular filaments might have a connexion with the overlying orbiculo-anterior capsular fibres was therefore considered.* Supporting

\footnotetext{
* It is generally accepted that the anterior zonular fibres terminate in sprays of minute fibrils
} in the peri-capsular membrane (Garzino, 1954). 
this idea is the similarity in staining and histochemical properties of these two structures: both show slight affinity for toluidine blue and other basic dyes; both exhibit increased affinity for certain basic dyes after preliminary oxidation with periodic acid or potassium permanganate; both are coloured by the aniline blue component of Mallory's connective tissue stain; and both give a positive reaction for polysaccharide with the periodic acid-Schiff technique. However, in spite of careful searching, no connexions between the zonular fibres and capsular filaments were found; they appear to be quite separate entities, and this is further supported by the fact that no capsular filaments are found behind the equator, although many zonular fibres are attached to the posterior lens capsule. The final verdict in this matter must perhaps await electron microscopy.

The possibility that the capsular filaments are an artefact has been considered and investigated as far as possible. The results are against such an interpretation, but it must be admitted that the inevitable trauma involved in making preparations and removing lenses has not been excluded.

Monahan, who studied the capsules from human senile cataractous lenses, noted that the number of visible channels varied inversely with the amount of cataract present, and that the channels were completely absent in total cataract. These observations and the results of his experiments on cataract production in animals led him to postulate blockage of the channels as the cause of senile cataract. In the present study no correlation between the number of filaments and the amount of cataract present was found; on the contrary these structures were present in apparently undiminished density and unchanged form in all thirty cataractous lenses examined. Moreover, they were apparently unchanged and appeared in normal density even at the hypermature stage of senile cataract.

Capsular filaments are visible in material fixed in Palade's solution and after subsequent embedding in methyl methacrylate, so that it may be possible to make further studies with the electron microscope-a method which was not available to me.

\section{Summary}

(1) Fine filaments, corresponding to previously described 'channels' in the lens capsules, have been studied.

(2) Their staining and histochemical properties were investigated, and a method for their demonstration is described.

(3) No relationship was found between these structures and the development of senile cataract.

(4) The problem of the nature of these filaments is discussed. 


\section{REFERENCES}

DAY, R. (1950). Amer. J. Ophthal., 33, 224.

FrançoIS, J., and RABAEY, M. (1958). Acta ophthal. (Kbh.), 36, 837.

FRIEDENWALD, J. S. (1930). Arch. Ophthal. (Chicago), 3, 182.

GARZINo, A. (1954). Rass. ital. Ottal., 23, 24.

GIFFORD, H., Jr. (1958). Amer. J. Ophthal., 46, 508.

GOMORI, G. (1941). Amer. J. Path., 17, 395.

Grignolo, A. (1954). G. ital. Oftal., 7, 300.

Косн, C. (1957). Zbl. ges. Ophthal., 72, 1.

KRAUSE, A. C. (1933). Arch. Ophthal. (Chicago), 9, 617.

MCCulloch, C. (1954). Trans. Amer. ophthal. Soc., 52, 525.

MonAHAN, R. H. (1953). Amer.J. Ophthal., 36, No. 6, part II, p. 24. (1958). Personal communication.

Palade, G. E. (1952). J. exp. Med., 95, 285.

Pirie, A., and van Heyningen, R. (1956). "Biochemistry of the Eye". Blackwell Scientific Publications, Oxford.

SalzmanN, M. (1912). "The Anatomy and Histology of the Human Eyeball", trans. E. V. L. Brown. University of Chicago Press, Chicago.

WislockI, G. B. (1952). Amer. J. Anat., 91, 233. 